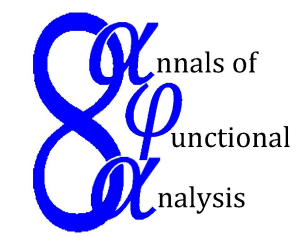

Ann. Funct. Anal. 6 (2015), no. 3, 8-28

http://doi.org/10.15352/afa/06-3-2

ISSN: 2008-8752 (electronic)

http://projecteuclid.org/afa

\title{
ON GENERALIZED WEIGHTED MEANS AND COMPACTNESS OF MATRIX OPERATORS
}

\author{
ALİ KARAİSA \\ Communicated by D. H. Leung
}

\begin{abstract}
This paper mainly divided into two parts. The first part gives same facts about topological properties of certain linear topological spaces, inclusion relations and matrix mappings. The second part establishes some identities or estimates for the matrix operator norms and the Hausdorff measures of noncompactness of certain matrix operators, characterize some classes of compact operators on these spaces.
\end{abstract}

\section{INTRODUCTION}

In the literature, by using the matrix domain over the paranormed spaces, many authors have defined new sequence spaces. Some of them as follows: Choudhary and Mishra [5] have studied sequence space $\overline{\ell(p)}$, where $\overline{\ell(p)}$ consists of the sequences whose $S$-trasforms are in $\ell(p)$, Başar and Altay [14] have defined the spaces $\lambda(u, v ; p)=\{\lambda(p)\}_{G}$ for $\lambda \in\left\{\ell_{\infty}, c_{0}, c\right\}$. The same authors also have defined the spaces [4] $r_{\infty}^{t}(p)=\left\{\ell_{\infty}(p)\right\}_{R^{t}}, r_{0}^{t}(p)=\left\{c_{0}(p)\right\}_{R^{t}}, r_{c}^{t}(p)=\{c(p)\}_{R^{t}}$. Recently, Karakaya et all. have introduced and studied the spaces [33] $\gamma(\lambda ; p)=$ $\{\gamma(p)\}_{\Lambda}$ for $\gamma \in\left\{\ell_{\infty}, c_{0}, c\right\} . R^{t}, G, \Lambda$ and $S$ denote Riesz, generalized difference, lambda and summation matrix, respectively. Also, the information on matrix domain of sequence spaces can be found (see [6, 7, 17, 19, 28]).

The main purpose of the present paper is to introduce the sequence spaces $\mu(u, v, p ; B)$ of non-absolute type and derive some related results. We also establish some inclusion relations. Furthermore, we determine the $\alpha-, \beta-$ and $\gamma$-duals of those spaces and construct their basis. Also, we characterize some classes of infinite matrices concerning the spaces $\mu(u, v, p ; B)$, where $\mu \in\left\{\ell_{\infty}, c_{0}, c\right\}$.

Date: Received: Jul. 8, 2014; Revised: Aug. 2, 2014; Accepted: Oct. 1, 2014.

2010 Mathematics Subject Classification. Primary 46A45; Secondary 40H05, $40 \mathrm{C} 05$.

Key words and phrases. BK-space, compactness, matrix operator, generalized weighted mean. 
Finally, we establish some identities or estimates for the operator norms and the Hausdorff measures of noncompactness of certain matrix operators on the spaces $c_{0}(u, v, p ; B), \ell_{\infty}(u, v, p ; B)$ and by using the Hausdorff measure of noncompactness, we characterize some classes of compact operators on these spaces.

\section{Notations and Auxiliary Facts}

By $\omega$, we shall denote the space of all real or complex valued sequences. Any vector subspace of $\omega$ is called sequence space. We shall write $\ell_{\infty}, c$ and $c_{0}$ for the spaces of all bounded, convergent and null sequence respectively. Also by $\ell_{1}$ and $\ell_{p}(1<p<\infty)$ we denote the spaces of all absolutely and $p$ - absolutely convergent series, respectively. Further, we shall write $b s$, cs for the spaces of all sequences associated with bounded and convergent series.

Let $\mu$ and $\gamma$ be two sequence spaces and $A=\left(a_{n k}\right)$ be an infinite matrix of real or complex numbers $a_{n k}$, where $n, k \in \mathbb{N}$. Then, we say that $A$ defines a matrix mapping from $\mu$ into $\gamma$, and we denote it by writing $A: \mu \rightarrow \gamma$, if for every sequence $x=\left(x_{k}\right) \in \mu$ the sequence $A x=\left\{(A x)_{n}\right\}$, the $A$-transform of $x$ is in $\gamma$; where

$$
(A x)_{n}=\sum_{k} a_{n k} x_{k} \quad(n \in \mathbb{N}) .
$$

The notation $(\mu: \gamma)$ denotes the class of all matrices $A$ such that $A: \mu \rightarrow \gamma$. Thus, $A \in(\mu: \gamma)$ if and only if the series on the right hand side of (2.1) converges for each $n \in \mathbb{N}$. The matrix domain $\mu_{A}$ of an infinite matrix $A$ in a sequence space $\mu$ is defined by

$$
\mu_{A}=\left\{x=\left(x_{k}\right) \in \omega: A x \in \mu\right\} .
$$

A linear topological space $X$ over the real field $\mathbb{R}$ is said to be a paranormed space if there exists subadditive function $h: X \longrightarrow \mathbb{R}$ such that $h(\theta)=0$, $h(-x)=h(x)$ and scalar multiplication is continuous, i.e., $\left|\alpha_{n}-\alpha\right| \longrightarrow 0$ and $h\left(x_{n}-x\right) \longrightarrow 0$ imply $h\left(\alpha_{n} x_{n}-\alpha x\right) \longrightarrow 0$ for all $\alpha$ 's in $\mathbb{R}$ and all $x$ 's in $X$, where $\theta$ is the zero vector in the linear space $X$.

Assume here and after that $p=\left(p_{k}\right)$ be a bounded sequence of strictly positive real numbers with $\sup p_{k}=H$ and $M=\max \{1, H\}$. Then, the linear spaces $\ell_{\infty}(p), c_{0}(p)$ and $c(p)$ were defined by Maddox [32](see also Simons [27] and Nakano [15] )

$$
\begin{aligned}
\ell_{\infty}(p) & =\left\{x=\left(x_{k}\right) \in \omega: \sup _{k \in \mathbb{N}}\left|x_{k}\right|^{p_{k}}<\infty\right\} \\
c_{0}(p) & =\left\{x=\left(x_{k}\right) \in \omega: \lim _{k \rightarrow \infty}\left|x_{k}\right|^{p_{k}}=0\right\} \\
c(p) & =\left\{x=\left(x_{k}\right) \in \omega: \exists l \in \mathbb{R} \text { such that } \lim _{k \rightarrow \infty}\left|x_{k}-l\right|^{p_{k}}=0\right\}
\end{aligned}
$$

which are the complete paranormed by

$$
h(x)=\sup _{k \in \mathbb{N}}\left|x_{k}\right|^{p_{k} / M} .
$$


Throughout the article, by $\mathcal{F}$ and $\mathbb{N}_{k}$, respectively, we denote the collection of all subsets of $\mathbb{N}$ and the set of all $n \in \mathbb{N}$ such that $n \geq k$. Also we write $e=(1,1,1, \ldots)$ and $e^{(n)}$ is the sequence whose only non-zero term is 1 in the $n^{t h}$ place for each $n \in \mathbb{N}$, where $\mathbb{N}=\{0,1,2, \ldots\}$.

Let $U$ denotes the set of all sequences $u=\left(u_{k}\right)$ such that $u_{k} \neq 0$ for all $k \in \mathbb{N}$ and $u, v \in U$, and define the matrix $G(u, v)=\left(g_{n k}\right)$ by

$$
g_{n k}=\left\{\begin{array}{cc}
u_{n} v_{k}, & (k<n), \\
u_{n} v_{n}, & (k=n), \\
0, & (k>n)
\end{array}\right.
$$

for all $k \in \mathbb{N}$, where $u_{n}$ depends only on $n$ and $v_{k}$ only on $k$. The matrix $G(u, v)$ which is called as generalized weighted mean or factorable matrix.

Let $r$ and $s$ be non-zero real numbers and define the generalized difference matrix $B(r, s)=\left\{b_{n k}(r, s)\right\}$ by

$$
b_{n k}(r, s)=\left\{\begin{array}{cc}
r, & k=n, \\
s, & k=n-1 \\
0, & \text { otherwise }
\end{array}\right.
$$

for all $n, k \in \mathbb{N}$. We note that the matrix $B(r, s)$ can be reduced to the difference matrix $\triangle$ in case $r=1$ and $s=-1$. So, the results related to the domain of the matrix $B(r, s)$ are more general and more comprehensive than the consequences of the domain of the matrix $\triangle$ and include them.

\section{Certain Main Results}

3.1. Basic Topological Properties. This part is devoted to examination of the basic topological properties of the sets $\mu(u, v, p ; B)$, where $\mu \in\left\{\ell_{\infty}, c_{0}, c\right\}$. For $u \in U$ and $\frac{1}{u}=\left(\frac{1}{u_{k}}\right)$.

Now, we introduce the new sequence spaces $\ell_{\infty}(u, v, p ; B), c_{0}(u, v, p ; B)$ and $c(u, v, p ; B)$ as follows:

$$
\begin{gathered}
\ell_{\infty}(u, v, p ; B)=\left\{x=\left(x_{k}\right) \in \omega: \sup _{n \in \mathbb{N}}\left|u_{n} \sum_{k=0}^{n} v_{k}\left(r x_{k}+s x_{k-1}\right)\right|^{p_{n}}<\infty\right\}, \\
c_{0}(u, v, p ; B)=\left\{x=\left(x_{k}\right) \in \omega: \lim _{n \rightarrow \infty}\left|u_{n} \sum_{k=0}^{n} v_{k}\left(r x_{k}+s x_{k-1}\right)\right|^{p_{n}}=0\right\}, \\
c(u, v, p ; B)=\left\{x=\left(x_{k}\right) \in \omega: \exists l \in \mathbb{R} \text { such that } \lim _{n \rightarrow \infty}\left|u_{n} \sum_{k=0}^{n} v_{k}\left(r x_{k}+s x_{k-1}\right)\right|^{p_{n}}=l\right\} .
\end{gathered}
$$

By the notation of $(2.2)$, we can redefine the spaces $\mu(u, v, p ; B)$ as follows:

$$
\mu(u, v, p ; B)=[\mu(u, v ; p)]_{B},
$$

where $B$ denotes the generalized difference matrix $B(r, s)=\left\{b_{n k}(r, s)\right\}$ defined by (2.3). On the other hand, we define the triangle matrix

$$
\widehat{G}(u, v, B)=G(u, v) B(r, s)=\left(\widehat{g}_{n k}\right)
$$




$$
\widehat{g}_{n k}=\left\{\begin{array}{cc}
u_{n}\left(r v_{k}+s v_{k+1}\right), & k<n, \\
r u_{n} v_{n}, & k=n, \\
0, & k>n
\end{array}\right.
$$

for all $n, k \in \mathbb{N}$. Then, we have following special cases.

(i) If $r=1$ and $s=-1$, then $\mu(u, v, p ; B)=\mu(u, v, p ; \triangle)$, where $\mu \in$ $\left\{\ell_{\infty}, c_{0}, c\right\}$ (see [3]).

(ii) Let $\lambda=\left(\lambda_{k}\right)$ is a strictly increasing sequence of positive reals tending to $\infty$. If $v=\left(\lambda_{k}-\lambda_{k-1}\right)$ and $u=\left(1 / \lambda_{n}\right)$ with $p=e$, then $c_{0}(u, v, p ; B)=$ $c_{0}^{\lambda}(B)$ and $c(u, v, p ; B)=c^{\lambda}(B)(\operatorname{see}[1])$.

(iii) If $v=\left(\lambda_{k}-\lambda_{k-1}\right)$ and $u=\left(1 / \lambda_{n}\right)$ with $p=e$ and $r=1, s=-1$, then $c_{0}(u, v, p ; \triangle)=c_{0}^{\lambda}(\Delta)$ and $c(u, v, p ; B)=c^{\lambda}(\Delta)($ see [24]).

(iv) If $v=\left(1+r^{k}\right)$ and $u=(1 / n+1)$ with $p=e$ and $r=1, s=-1$, then $c_{0}(u, v, p ; B)=a_{0}^{r}(\triangle)$ and $c(u, v, p ; B)=a_{c}^{r}(\Delta)$ (see [9]).

(v) If $v=\left(q_{k}\right)$ and $u=\left(1 / \sum_{k=0}^{n} q_{k}\right)$, then $\ell_{\infty}(u, v, p ; B)=r_{\infty}^{q}(p, B)$ and $c_{0}(u, v, p ; B)=r_{0}^{q}(p, B)$ as well as $c(u, v, p ; B)=r_{c}^{q}(p, B)$ (see [18]).

Define the sequence $y=\left(y_{k}\right)$, which will be frequently used, as the $\widehat{G}$-transform of a sequence $x=\left(x_{k}\right)$, i.e.

$$
y_{k}=\sum_{i=0}^{k} u_{k} v_{i}\left(r x_{i}+s x_{i-1}\right)
$$

and every $x=\left(x_{k}\right) \in \omega$ which leads us together with (2.2) to the fact that

$$
\mu(u, v, p ; B)=[\mu(p)]_{\widehat{G}} .
$$

Also we derive that equality of (3.1)

$$
y_{0}=r u_{0} v_{0} \text { and } y_{k}=u_{k}\left(\sum_{i=0}^{k-1}\left(r v_{i}+s v_{i+1}\right) x_{i}+r u_{k} v_{k} x_{k}\right) \text { for all } k \geq 1 \text {. }
$$

Theorem 3.1. We have the following

(a): $\mu(u, v, p ; B)$ is the complete linear metric space paranormed by $g$, defined by

$$
g(x)=\sup _{n}\left|\sum_{k=0}^{n-1} u_{n}\left(r v_{k}+s v_{k+1}\right) x_{k}+u_{n} v_{n} x_{n}\right|^{\frac{p_{n}}{M}}
$$

where $M=\max \left\{1, \sup p_{n}\right\}$ and $0<p_{n} \leq H<\infty$ for all $n \in \mathbb{N}$.

(b): Then, $\mu(u, v, p ; B)=\mu_{\widehat{G}}$ is a $B K$-space with the norm $\|x\|_{\mu(u, v, p ; B)}=$ $\|\widehat{G} x\|_{\infty}$. That is,

$$
\|x\|_{\mu(u, v, p ; B)}=\sup _{n}\left|(\widehat{G} x)_{n}\right| .
$$


Proof. (a) We prove the theorem only for the space $c_{0}(u, v, p ; B)$. The linearity of $c_{0}(u, v, p ; B)$ with respect to the coordinatewise addition and scalar multiplication follows from the following inequalities which are satisfied for $z, x \in c_{0}(u, v, p ; B)$ $[25$, p.30]

$$
\begin{aligned}
& \sup _{n}\left|\sum_{k=0}^{n-1} u_{n}\left(r v_{k}+s v_{k+1}\right)\left(x_{k}+z_{k}\right)+u_{n} v_{n}\left(x_{n}+z_{n}\right)\right|^{\frac{p_{n}}{M}} \\
\leq & \sup _{n}\left|\sum_{k=0}^{n-1} u_{n}\left(r v_{k}+s v_{k+1}\right) x_{k}+u_{n} v_{n} x_{n}\right|^{\frac{p_{n}}{M}} \\
& +\sup _{n}\left|\sum_{k=0}^{n-1} u_{n}\left(r v_{k}+s v_{k+1}\right) z_{k}+u_{n} v_{n} z_{n}\right|^{\frac{p_{n}}{M}} .
\end{aligned}
$$

For any $\alpha \in \mathbb{R}$ (see[32]), we get

$$
|\alpha|^{p_{k}} \leq \max \left\{1,|\alpha|^{M}\right\} .
$$

It is clear that $g(\theta)=0$ and $g(x)=g(-x)$ for all $x \in c_{0}(u, v, p ; B)$. The inequalities (3.3) and (3.4) again yield the subadditivity of $g$ and

$$
g(\alpha x) \leq \max \left\{1,|\alpha|^{M}\right\} g(x) .
$$

Let $\left\{x^{n}\right\}$ be any sequence of points in $c_{0}(u, v, p ; B)$ such that $g\left(x^{n}-x\right) \longrightarrow 0$ and $\left(\alpha_{n}\right)$ also be any sequence of scalars that $\alpha_{n} \longrightarrow \alpha$. Then, we obtain

$$
g\left(\alpha_{n} x^{n}-\alpha x\right) \leq g\left[\left(\alpha_{n}-\alpha\right)\left(x^{n}-x\right)\right]+g\left[\alpha\left(x^{n}-x\right)\right]+g\left[x\left(\alpha^{n}-\alpha\right)\right] .
$$

It follows from $\alpha_{n} \longrightarrow \alpha$ as $n \longrightarrow \infty$ that $\left|\alpha_{n}-\alpha\right|<1$ for all sufficient large $n$. Therefore,

$$
\lim _{n \rightarrow \infty} g\left[\left(\alpha_{n}-\alpha\right)\left(x^{n}-x\right)\right] \leq \lim _{n \rightarrow \infty} g\left[\left(x^{n}-x\right)\right]=0 .
$$

By (3.5), we have

$$
\lim _{n \rightarrow \infty} g\left[\alpha\left(x^{n}-x\right)\right] \leq \max \left\{1,|\alpha|^{M}\right\} \lim _{n \rightarrow \infty} g\left[\left(x^{n}-x\right)\right]=0 .
$$

Furthermore, we get

$$
\lim _{n \rightarrow \infty} g\left[x\left(\alpha^{n}-\alpha\right)\right] \leq \lim _{n \rightarrow \infty}\left|\alpha_{n}-\alpha\right| g(x)=0 .
$$

Then, we obtain from (3.6)-(3.9) that $g\left(\alpha_{n} x^{n}-\alpha x\right) \longrightarrow 0$ as $n \longrightarrow \infty$. This shows that $g$ is a paranorm on $c_{0}(u, v, p ; B)$.

It remains to prove the completeness of the space $c_{0}(u, v, p ; B)$. Let $\left\{x^{j}\right\}$ be any Cauchy sequence in the space $c_{0}(u, v, p ; B)$, where $x^{i}=\left(x_{0}^{i}, x_{2}^{i}, x_{2}^{i}, \ldots\right)$. Then for a given $\varepsilon$ there exists a positive integer $n_{0}(\varepsilon)$ such that

$$
g\left(x^{j}-x^{i}\right)<\varepsilon
$$

for all $i, j \geq n_{0}(\varepsilon)$. Using definition of $g$, we obtain for each fixed $n \in \mathbb{N}$ that

$$
\left|\left(\widehat{G} x^{j}\right)_{n}-\left(\widehat{G} x^{i}\right)_{n}\right|^{\frac{p_{n}}{M}} \leq \sup _{n}\left|\left(\widehat{G} x^{j}\right)_{n}-\left(\widehat{G} x^{i}\right)_{n}\right|^{\frac{p_{n}}{M}}<\frac{\varepsilon}{2}
$$


for every $i, j \geq n_{0}(\varepsilon)$, which lead us to fact that $\left\{\left(\widehat{G} x^{0}\right)_{k},\left(\widehat{G} x^{1}\right)_{k},\left(\widehat{G} x^{2}\right)_{k}, \ldots\right\}$ is a Cauchy sequence of real numbers for every fixed $n \in \mathbb{N}$. Since $\mathbb{R}$ is complete, it converges, say $\left(\widehat{G} x^{j}\right)_{k} \longrightarrow(\widehat{G} x)_{k}$ as $j \longrightarrow \infty$. Using these infinitely many limits, we may write the sequence $\left\{(\widehat{G} x)_{0},(\widehat{G} x)_{1},(\widehat{G} x)_{2}, \ldots\right\}$. Using $(3.10)$ as $i \longrightarrow \infty$ and for all $j \geq n_{0}(\varepsilon)$, we have

$$
\left|\left(\widehat{G} x^{j}\right)_{n}-(\widehat{G} x)_{n}\right|<\frac{\varepsilon}{2}
$$

for every fixed $n \in \mathbb{N}$. Since $x^{j}=\left(x_{k}^{(j)}\right) \in c_{0}(u, v, p ; B)$ for each $j \in \mathbb{N}$, there exists $n_{0}(\varepsilon)$ such that $\left|\left(\widehat{G} x^{j}\right)_{n}\right|^{\frac{p_{n}}{M}}<\frac{\varepsilon}{2}$ for every $j \geq n_{0}(\varepsilon)$. We obtain by (3.11)

$$
\left|\left(\widehat{G} x^{j}\right)_{n}\right|^{\frac{p_{n}}{M}} \leq\left|\left(\widehat{G} x^{j}\right)_{n}-(\widehat{G} x)_{n}\right|^{\frac{p_{n}}{M}}+\left|\left(\widehat{G} x^{j}\right)_{n}\right|^{\frac{p_{n}}{M}}<\varepsilon
$$

for every $j \geq n_{0}(\varepsilon)$. Thus, we get $x \in c_{0}(u, v, p ; B)$. Since $\left(x^{j}\right)$ was an arbitrary Cauchy sequence, the space $c_{0}(u, v, p ; B)$ is complete.

(b) Since the sequence space $\mu$ endowed with the norm $\|\cdot\|_{\infty}$ is $B K$-space (see[26, Example 7.3.2(b),(c)]) and the matrix $\widehat{G}$ is a triangle, Theorem 4.3 .2 of Wilansky [2, p.61] gives the fact that the spaces $\mu(u, v, p ; B)$ are $B K$-space with the norm in (3.2).

One can easily check that the absolute property does not hold on the space $\mu(u, v, p ; B)$, that is $\|x\|_{\mu(u, v, p ; B)} \neq\|\mid x\|_{\mu(u, v, p ; B)}$ for at least one sequence in the space $\mu(u, v, p ; B)$ and this tells us that $\mu(u, v, p ; B)$ none-absolute type, where $|x|=\left(\left|x_{k}\right|\right)$.

Theorem 3.2. The sequence spaces $\ell_{\infty}(u, v, p ; B), c_{0}(u, v, p ; B)$ and $c(u, v, p ; B)$ of none-absolute type is linearly isomorphic to the spaces $\ell_{\infty}(p), c_{0}(p)$ and $c(p)$, respectively, where $0<p_{k} \leq H<\infty$.

Proof. To prove the fact that $c_{0}(u, v, p ; B) \cong c_{0}(p)$ we should show the existence of a linear bijection between the spaces $c_{0}(u, v, p ; B)$ and $c_{0}(p)$. Consider the transformation $T$ defined with the notation of (2.2) from $c_{0}(u, v, p ; B)$ to $c_{0}(p)$ by $x \mapsto y=T x=\widehat{G} x$. The linearity of $T$ is clear. Further, it is clear that $x=\theta$ whenever $T x=\theta$ and hence $T$ is injective.

Let $y=\left(y_{k}\right) \in c_{0}(p)$ and define the sequence $x=\left(x_{k}\right)$ by

$$
x_{k}=\sum_{j=0}^{k-1}\left(\frac{-s}{r}\right)^{k-j}\left[\frac{1}{r v_{j}}+\frac{1}{s v_{j+1}}\right] \frac{1}{u_{j}} y_{j}+\frac{1}{r u_{k} v_{k}} y_{k} \text { for each } k \in \mathbb{N} .
$$

Then, we have

$$
g(x)=\sup _{n}\left|\sum_{k=0}^{n-1} u_{n}\left(r v_{k}+s v_{k+1}\right) x_{k}+r u_{n} v_{n} x_{n}\right|^{\frac{p_{n}}{M}}=\sup _{n}\left|y_{n}\right|^{\frac{p_{n}}{M}}=h(y) .
$$

Therefore, we have $x \in c_{0}(u, v, p ; B)$. As a result, $T$ is surjective. This implies $T$ is linear bijection. So, the space $c_{0}(u, v, p ; B)$ and $c_{0}(p)$ are linearly isomorphic as desired. This completes the proof. 
Now, we define the Schauder basis of a paranormed sequence space and then give the basis of the sequence spaces $c(u, v, p ; B)$ and $c_{0}(u, v, p ; B)$. If a sequence space $\gamma$ paranormed by $g_{1}$ contains a sequence $\left(b_{k}\right)$ with the property that for every $x \in \gamma$, there is a unique of scalars $\left(\alpha_{k}\right)$ such that

$$
\lim _{n \rightarrow \infty} g_{1}\left(x-\sum_{k=0}^{n} \alpha_{k} b_{k}\right)=0,
$$

then $\left(b_{k}\right)$ is called a Schauder basis (or briefly basis) for $\gamma$. The series $\sum \alpha_{k} b_{k}$ which has the sum $x$ is called the expansion of $x$ with respect to $\left(b_{k}\right)$, and written as $x=\sum \alpha_{k} b_{k}$. It is known from Theorem 2.3 of Jarrah and Malkowsky [22] that the domain $\nu_{T}$ of an infinite matrix $T$ in a sequence space $\nu$ has a basis if and only if $\nu$ has a basis, if $T$ is a triangle. As a direct consequence of this fact, we have:

Corollary 3.3. Let $\alpha_{k}=(\widehat{G} x)_{k}$ for all $k \in \mathbb{N}$ and $l=\lim _{k \rightarrow \infty}(\widehat{G} x)_{k}$. Define the sequence $b^{(k)}=\left\{b_{n}^{(k)}\right\}$ for every fixed $n \in \mathbb{N}$ by

$$
b_{n}^{(k)} \begin{cases}\left(\frac{-s}{r}\right)^{n-k}\left[\frac{1}{r v_{k}}+\frac{1}{s v_{k+1}}\right] \frac{1}{u_{k},}, & k<n, \\ \frac{1}{r u_{n} v_{n}}, & k=n, \\ 0, & k>n\end{cases}
$$

Then, the following statements hold.

(a) The sequence $\left(b_{n}^{(k)}\right)$ is a basis for the space $c_{0}(u, v, p ; B)$ and every $x \in$ $c_{0}(u, v, p ; B)$ has a unique representation of the form $x=\sum_{k} \alpha_{k} b^{(k)}$.

(b) The sequence space $\left\{b, b^{(0)}, b^{(1)}, \ldots\right\}$ is a basis for the space $c(u, v, p ; B)$ and any $x \in c(u, v, p ; B)$ has a unique representation of the for $x=l b+\sum_{k}\left[\alpha_{k}-l\right] b_{k}$, where

$$
b=\left(b_{k}\right)=\left\{\sum_{j=0}^{k-1}\left(\frac{-s}{r}\right)^{k-j}\left[\frac{1}{r v_{j}}+\frac{1}{s v_{j+1}}\right] \frac{1}{u_{j}}+\frac{1}{r u_{k} v_{k}}\right\}_{k=0}^{\infty} .
$$

3.2. Inclusion Relations. In this part, we give some inclusion relations concerning the spaces $\mu(u, v, p ; B)$.

Theorem 3.4. The inclusions $c_{0}(u, v, p ; B) \subset c(u, v, p ; B) \subset \ell_{\infty}(u, v, p ; B)$ strictly hold.

Proof. Let $x=\left(x_{k}\right) \in c_{0}(u, v, p ; B)$. This implies $\widehat{G} x \in c_{0}(p)$. Then, since the inclusion $c_{0}(p) \subset c(p)$ holds, $\widehat{G} x \in c(p)$ which means that $x \in c(u, v, p ; B)$. Further consider the sequence as follows:

$x_{k}=\frac{1}{2}\left[\sum_{j=0}^{k-1}\left(\frac{-s}{r}\right)^{k-j}\left[\frac{1}{r v_{j}}+\frac{1}{s v_{j+1}}\right] \frac{1}{u_{j}}+\frac{1}{r u_{k} v_{k}}\right], p_{n}=\frac{2 n+5}{n+1}$ for each $n \in \mathbb{N}$.

Then, we have

$$
\left|\widehat{G}_{n}(x)\right|^{p_{n}}=\frac{1}{2^{\frac{2 n+5}{n+1}}} .
$$


From (3.13), we get $x$ is in $c(u, v, p ; B)$ but not in $c_{0}(u, v, p ; B)$. Since the inclusion $c(p) \subset \ell_{\infty}(p)$ is strict, one can find at least a sequence $\widehat{G} x \in \ell_{\infty}(p) \backslash c(p)$ which shows that $\left.\ell_{\infty}(u, v, p ; B) \backslash c(u, v, p ; B)\right)$ is not empty, as desired.

Theorem 3.5. Following statements are hold.

(i): If $p_{n}>1$ for all $n \in \mathbb{N}$, then the inclusion $\mu(u, v, B) \subset \mu(u, v, p ; B)$ holds

(ii): If $p_{n}<1$ for all $n \in \mathbb{N}$, then the inclusion $\mu(u, v, p ; B) \subset \mu(u, v, B)$ holds

where $\mu \in\left\{\ell_{\infty}, c_{0}, c\right\}$.

Proof. ( $i$ ) If $p_{n}=p$ for all $n \in \mathbb{N}$, then we can write $c_{0}(u, v, B)$ instead of $c_{0}(u, v, p ; B)$. Let $x \in c_{0}(u, v, B)$. It is clear that $\widehat{G} x \in c_{0}$. We can find $m \in \mathbb{N}$ such that $|\widehat{G} x|<1$ for all $n \geq m$. By our assumption $(i)$, we have $|\widehat{G} x|^{p_{n}}<|\widehat{G} x|$ for all $n \geq m$. Therefore, we get $x \in c_{0}(u, v, p ; B)$.

(ii) Let $x \in c_{0}(u, v, p ; B)$. Then $\widehat{G} x \in c_{0}(p)$ and there exists $m \in \mathbb{N}$ such that $|\widehat{G} x|^{p_{n}}<1$ for all $n \geq m$. Now, consider following inequality:

$$
|\widehat{G} x|=\left(|\widehat{G} x|^{p_{n}}\right)^{1 / p_{n}}<|\widehat{G} x|^{p_{n}}
$$

for all $n \geq m$. So we have $x \in c_{0}(u, v, B)$. This completes the proof.

3.3. Duals. In this part, we state and prove certain theorems to determine the $\alpha-, \beta-$ and $\gamma$ duals of the spaces $\mu(u, v, p ; B)$ for $\mu \in\left\{\ell_{\infty}, c_{0}, c\right\}$. We start with the definition of the alpha, beta and gamma duals. If $x$ and $y$ are sequences and $X$ and $Y$ are subsets of $\omega$, then we write $x \cdot y=\left(x_{k} y_{k}\right)_{k=0}^{\infty}, x^{-1} * Y=\{a \in \omega$ : $a \cdot x \in Y\}$ and

$$
M(X, Y)=\bigcap_{x \in X} x^{-1} * Y=\{a: a \cdot x \in Y \text { for all } x \in X\}
$$

for the multiplier space of $X$ and $Y$. One can easily observe for a sequence space $Z$ with $Y \subset Z$ and $Z \subset X$ that inclusions $M(X, Y) \subset M(X, Z)$ and $M(X, Y) \subset M(Z, Y)$ hold respectively. The $\alpha-, \beta$-and $\gamma$-duals of a sequence space, which are respectively denoted by $X^{\alpha}, X^{\beta}$ and $X^{\gamma}$ are defined by

$$
X^{\alpha}=M\left(X, \ell_{1}\right), X^{\beta}=M(X, c s) \text { and } X^{\gamma}=M(X, b s) .
$$

It is obvious that $X^{\alpha} \subset X^{\beta} \subset X^{\gamma}$. Also, it can easily be seen that the inclusions $X^{\alpha} \subset Y^{\alpha}, X^{\beta} \subset Y^{\beta}$ and $X^{\gamma} \subset Y^{\gamma}$ hold whenever $Y \subset X$.

Theorem 3.6. Define the matrix $D=\left(d_{n k}\right)$ by

$$
d_{n k}= \begin{cases}\left(\frac{-s}{r}\right)^{n-k}\left[\frac{1}{r v_{k}}+\frac{1}{s v_{k+1}}\right] \frac{1}{u_{k}} a_{n}, & k<n, \\ \frac{1}{r u_{n} v_{n}} a_{n}, & k=n, \\ 0, & k>n\end{cases}
$$

for all $n, k \in \mathbb{N}$. Then,

$$
\mu^{\alpha}(u, v, p ; B)=\left\{a=\left(a_{k}\right) \in w: D \in\left(\mu(p): \ell_{1}\right)\right\}
$$


Proof. Let $a=\left(a_{n}\right) \in w$. Then by using (3.12), we immediately derive for every $n \in \mathbb{N}$ that

$$
a_{n} x_{n}=\frac{1}{r} \sum_{k=0}^{n}\left(\frac{-s}{r}\right)^{n-k}\left[\frac{1}{r v_{k}}+\frac{1}{s v_{k+1}}\right] \frac{1}{u_{k}} y_{k} a_{n}+\frac{1}{r u_{n} v_{n}} y_{n} a_{n}=(D y)_{n} .
$$

Thus, we observe by (3.14) that $a x=\left(a_{n} x_{n}\right) \in \ell_{1}$ whenever $x=\left(x_{k}\right) \in$ $\mu(u, v, p ; B)$ if and only if $D_{n}(y) \in \ell_{1}$ whenever $y=\left(y_{k}\right) \in \mu(p)$ which implies $a=\left(a_{k}\right) \in\{\mu(u, v, p ; B)\}^{\alpha}$ if and only if $D \in\left(\mu(p): \ell_{1}\right)$.

Theorem 3.6 corresponds in the special case $q_{n}=1$ for all $n \in \mathbb{N}$ to Part (1-3) of Theorem 5.1 of [16].

As a direct consequence of Theorem 3.6, we have following.

Corollary 3.7. Let $K^{*}=K \cap\{n \in \mathbb{N}: n-1 \leq k \leq n\}$ for $K \subset \mathcal{F}$ and $M \in \mathbb{N}_{2}$. Define the sets $t_{1}(p), t_{2}(p), t_{3}(p)$ as follows:

$$
\begin{aligned}
& t_{1}(p):=\bigcap_{M>1}\left\{a=\left(a_{k}\right) \in \omega: \sup _{K \in \mathcal{F}} \sum_{n}\left|\sum_{k \in K^{*}} d_{n k} M^{1 / p_{k}}\right|<\infty\right\}, \\
& t_{2}(p):=\bigcup_{M>1}\left\{a=\left(a_{k}\right) \in \omega: \sum_{n}\left|\sum_{k} d_{n k}\right|<\infty\right\}, \\
& t_{3}(p):=\bigcup_{M>1}\left\{a=\left(a_{k}\right) \in \omega: \sup _{K \in \mathcal{F}} \sum_{n}\left|\sum_{k \in K^{*}} d_{n k} M^{-1 / p_{k}}\right|<\infty\right\} .
\end{aligned}
$$

Then, $\ell_{\infty}^{\alpha}(u, v, p ; B)=t_{1}(p), c_{0}^{\alpha}(u, v, p ; B)=t_{3}(p)$ and $c^{\alpha}(u, v, p ; B)=t_{2}(p) \cap$ $t_{3}(p)$.

Theorem 3.8. Let $u, v \in U$ and $r, s$ be non-zero real numbers. Define the matrix $C=\left(c_{n k}\right)$ by

$$
c_{n k}= \begin{cases}\widehat{a}_{k}(n), & 0 \leq k \leq n-1 \\ \frac{1}{r u_{n} v_{n}} a_{n}, & k=n \\ 0, & k>n\end{cases}
$$

for all $n, k \in \mathbb{N}$, where

$$
\begin{gathered}
\widehat{a}_{k}(n)=\frac{1}{u_{k}}\left[\frac{a_{k}}{r v_{k}}+\left(\frac{1}{r v_{k}}+\frac{1}{s v_{k+1}}\right) \sum_{j=k+1}^{n}\left(\frac{-s}{r}\right)^{k-j} a_{j}\right] \text { for } k<n . \\
\mu^{\beta}(u, v, p ; B)=\left\{a=\left(a_{k}\right) \in w: C \in(\mu(p): c)\right\}, \\
\mu^{\gamma}(u, v, p ; B)=\left\{a=\left(a_{k}\right) \in w: C \in\left(\mu(p): \ell_{\infty}\right)\right\} .
\end{gathered}
$$


Proof. Let us consider following equation

$$
\begin{aligned}
\sum_{k=0}^{n} a_{k} x_{k} & =\sum_{k=0}^{n}\left\{\sum_{j=0}^{k-1}\left(\frac{-s}{r}\right)^{k-j}\left[\frac{1}{r v_{j}}+\frac{1}{s v_{j+1}}\right] \frac{1}{u_{j}} y_{j}+\frac{1}{u_{k} v_{k}} y_{k}\right\} a_{k} \\
& =\sum_{k=0}^{n-1} \frac{1}{u_{k}}\left[\frac{a_{k}}{r v_{k}}+\left(\frac{1}{r v_{k}}+\frac{1}{s v_{k+1}}\right) \sum_{j=k+1}^{n}\left(\frac{-s}{r}\right)^{k-j} a_{j}\right] y_{k}+\frac{a_{n}}{r u_{n} v_{n}} y_{n} \\
& =\sum_{k=0}^{n-1} \widehat{a}_{k}(n) y_{k}+\frac{a_{n}}{r u_{n} v_{n}} y_{n} \\
& =(C y)_{n} \text { for all } n \in \mathbb{N},
\end{aligned}
$$

where $C=\left(c_{n k}\right)$ defined by (3.15). We deduce from (3.16) that $a x=\left(a_{n} x_{n}\right) \in c s$ or $b s$ whenever $x=\left(x_{k}\right) \in \mu(u, v, p ; B)$ if and only if $C y \in c$ or $\ell_{\infty}$ whenever $y=\left(y_{k}\right) \in \mu(p)$. This means that $a=\left(a_{k}\right) \in\{\mu(u, v, p ; B)\}^{\beta}$ or $a=\left(a_{k}\right) \in$ $\{\mu(u, v, p ; B)\}^{\gamma}$ if and only if $C \in(\mu(p): c)$ or $C \in\left(\mu(p): \ell_{\infty}\right)$. This completes the proof.

As a direct consequence of Theorem 3.8, we have following.

Corollary 3.9. Define the sets $d_{1}(p), d_{2}(p), d_{3}(p), d_{4}(p)$ and $d_{5}(p)$ as follows:

$$
\begin{aligned}
& d_{1}(p)=\bigcap_{B>1}\left\{a=\left(a_{k}\right) \in \omega: \sum_{k}\left|\widehat{a}_{k}(n)\right| B^{1 / p_{k}} \text { convergent uniformly in } n\right\}, \\
& d_{2}(p)=\left\{a=\left(a_{k}\right) \in \omega:\left(\frac{1}{r u_{n} v_{n}} a_{k} B^{1 / p_{k}}\right) \in c_{0}\right\}, \\
& d_{3}(p)=\bigcap_{B>1}\left\{a=\left(a_{k}\right) \in \omega: \sum_{k}\left|\widehat{a}_{k}(n)\right| B^{1 / p_{k}}<\infty\right\}, \\
& d_{4}(p)=\bigcup_{B>1}\left\{a=\left(a_{k}\right) \in \omega: \sum_{k}\left|\widehat{a}_{k}(n)\right| B^{-1 / p_{k}}<\infty\right\}, \\
& d_{5}(p)=\bigcap_{B>1}\left\{a=\left(a_{k}\right) \in \omega:\left\{\widehat{a}_{k}(n)\right\} B^{1 / p_{k}} \in \ell_{\infty}\right\},
\end{aligned}
$$

$\left\{\ell_{\infty}(u, v, p ; B)\right\}^{\beta}=d_{1}(p) \cap d_{2}(p),\left\{c_{0}(u, v, p ; B)\right\}^{\beta}=\left\{c_{0}(u, v, p ; B)\right\}^{\gamma}=d_{4}(p)$, $\{c(u, v, p ; B)\}^{\beta}=d_{4}(p) \cap c s,\{c(u, v, p ; B)\}^{\gamma}=d_{4}(p) \cap b s$ and $\left\{\ell_{\infty}(u, v, p ; B)\right\}^{\gamma}=$ $d_{3}(p) \cap d_{5}(p)$.

3.4. Certain Matrix Mappings. In this part, we characterize the matrix mappings from the sequence space $\mu(u, v, p ; B)$ into any given sequence space, where $\mu \in\left\{c_{0}, c, \ell_{\infty}\right\}$. For an infinite matrix $A=\left(a_{n k}\right)$, we write for brevity that

$$
\bar{a}_{n k}(m)=\frac{1}{u_{k}}\left[\frac{a_{n k}}{r v_{k}}+\left(\frac{1}{r v_{k}}+\frac{1}{s v_{k+1}}\right) \sum_{j=k+1}^{m}\left(\frac{-s}{r}\right)^{k-j} a_{n j}\right] \quad(k<m)
$$


and

$$
\bar{a}_{n k}=\frac{1}{u_{k}}\left[\frac{a_{n k}}{r v_{k}}+\left(\frac{1}{r v_{k}}+\frac{1}{s v_{k+1}}\right) \sum_{j=k+1}^{\infty}\left(\frac{-s}{r}\right)^{k-j} a_{n j}\right],
$$

for all $k, n, m \in \mathbb{N}$ provided the convergence of the series. Now, we give the characterization of the classes $(\mu(u, v, p ; B): \nu)$ and $(\nu: \mu(u, v, p ; B))$, where $\nu$ any given sequence space.

Theorem 3.10. $A=\left(a_{n k}\right) \in(\mu(u, v, p ; B): \nu)$ if and only if $D=\left(d_{n k}\right) \in(\mu(p)$ : v) and

$$
E^{(n)} \in(\mu(p): c)
$$

for every fixed $n \in \mathbb{N}$, where $d_{n k}=\bar{a}_{n k}$ and $E^{(n)}=\left(e_{m k}^{(n)}\right)$ with

$$
e_{m k}^{n}= \begin{cases}\bar{a}_{n k}(m), & 0 \leq k \leq m-1, \\ \frac{1}{r u_{m} v_{m}} a_{m k}, & k=m, \\ 0, & k>m\end{cases}
$$

for all $k, n, m \in \mathbb{N}$.

Proof. Assume that $\nu$ is any given sequence space and keep in mind that the spaces $\mu(u, v, p ; B)$ and $\mu(p)$ are paranorm isomorphic.

Let $A=\left(a_{n k}\right) \in(\mu(u, v, p ; B): \nu)$ and $x \in \mu(u, v, p ; B)$. Then we obtain the equality

$$
\sum_{k=0}^{m} a_{n k} x_{k}=\sum_{k=0}^{m-1} \bar{a}_{n k}(m) y_{k}+\frac{a_{n m}}{r u_{m} v_{m}} y_{m}=\sum_{k=0}^{m} e_{m k}^{n} y_{k}
$$

for all $m, n \in \mathbb{N}$. Since $A x$ exists, $E^{(n)}$ must belong to the class $(\mu(p): c)$. Letting $m \longrightarrow \infty$ in the equality (3.19) we have that $A x=D y$. Since $A x \in \nu$, then $D y \in \nu$. That is $D=\left(d_{n k}\right) \in(\mu(p): \nu)$.

Conversely, let $D \in(\mu(p): \nu)$ and (3.18) holds, and take any $x \in \mu(u, v, p ; B)$. Then, we have $\left(d_{n k}\right)_{k \in \mathbb{N}} \in \mu^{\beta}(p)$ which gives together with (3.18) that $\left(a_{n k}\right)_{k \in \mathbb{N}} \in$ $[\mu(u, v, p ; B)]^{\beta}$ for each $n \in \mathbb{N}$. Thus, $A x$ exists and we obtain from equality (3.19) as $m \longrightarrow \infty$ that $D y=A x$ and which means that $A=\left(a_{n k}\right) \in(\mu(u, v, p ; B): \nu)$. This completes the proof.

Theorem 3.11. Suppose that the entries of the infinite matrices $A=\left(a_{n k}\right)$ and $B=\left(b_{n k}\right)$ are connected with the relation

$$
b_{n k}=\sum_{j=0}^{n-1}\left(r v_{j}+s v_{j+1}\right) a_{j k}+r u_{n} v_{n} a_{n k}
$$

for all $k, n \in \mathbb{N}$ and $\nu$ be any given sequence space. Then, $A \in(\nu: \mu(u, v, p ; B))$ if and only if $B \in(\nu: \mu(p))$. 
Proof. Let $x=\left(x_{k}\right) \in \nu$ and consider the equality

$$
\begin{aligned}
\sum_{k=0}^{m} b_{n k} x_{k} & =\sum_{k=0}^{m}\left(\sum_{j=0}^{n-1}\left(r v_{j}+s v_{j+1}\right) a_{j k}+r u_{n} v_{n} a_{n k}\right) x_{k} \\
& =\sum_{j=0}^{n-1}\left(r v_{j}+s v_{j+1}\right) \sum_{k=0}^{m} a_{j k} x_{k}+r u_{n} v_{n} \sum_{k=0}^{m} a_{n k} x_{k}
\end{aligned}
$$

for all $k, m, n \in \mathbb{N}$ which yields as $m \rightarrow \infty$ that $B x=\widehat{G}(A x)$. Therefore, $A x \in \mu(u, v, p ; B)$ whenever $x \in \nu$ if and only if $B x \in \mu(p)$ whenever $x \in \nu$. This step completes the proof.

The necessary and sufficient conditions characterizing the matrix mapping between the sequence spaces $\ell_{\infty}(p), c_{0}(p)$ and $c(p)$ of Maddox are determined by Grosse-Erdmann [16]. Let $N$ and $K$ denote subsets of $\mathbb{N}, L$ and $M$ also denote the natural numbers and define the sets $K_{1}$ and $K_{2}$ by $K_{1}=\left\{k \in \mathbb{N}: p_{k} \leq 1\right\}$, $K_{2}=\left\{k \in \mathbb{N}: p_{k}>1\right\}$. Before giving the theorems, let us suppose that $\left(q_{n}\right)$ is non-decreasing sequence of positive real numbers and consider following condition:

$$
\begin{aligned}
& \exists M, \quad \sup _{K} \sum_{n}\left|\sum_{k \in K} a_{n k} M^{-1 / p_{k}}\right|^{q_{n}}<\infty \quad\left(q_{n} \geq 1\right), \\
& \sum_{n}\left|\sum_{k} a_{n k}\right|^{q_{n}}<\infty, \quad\left(q_{n} \geq 1\right), \\
& \forall M, \quad \sup _{K} \sum_{n}\left|\sum_{k \in K} a_{n k} M^{-1 / p_{k}}\right|^{q_{n}}<\infty \quad\left(q_{n} \geq 1\right), \\
& \lim _{n \rightarrow \infty}\left|a_{n k}\right|^{q_{n}}=0 \quad \text { for all } k, \\
& \forall L, \quad \sup _{n \in \mathbb{N}} \sup _{k \in K_{1}}\left|a_{n k} L^{1 / q_{n}}\right|^{p_{k}}<\infty, \\
& \forall L, \quad \exists M, \quad \sup _{n \in K_{1}} \sum_{k \in K_{2}}\left|a_{n k} L^{1 / q_{n}} M^{-1}\right|^{p_{k}^{\prime}}<\infty, \\
& \forall L, \quad \exists M, \quad \sup _{n \in \mathbb{N}} L^{1 / q_{n}} \sum_{k}\left|a_{n k}\right| M^{-1}<\infty, \\
& \lim _{n \rightarrow \infty}\left|\sum_{k} a_{n k}\right|^{q_{n}}=0 \\
& \forall M, \quad \lim _{n \rightarrow \infty}\left(\sum_{k}\left|a_{n k}\right| M^{1 / p_{k}}\right)^{q_{n}}=0, \\
& \sup \sup _{n \in \mathbb{N}}\left|a_{n \in}\right|_{k \in K_{1}}^{p_{k}},
\end{aligned}
$$




$$
\begin{aligned}
& \exists M, \quad \sup _{k \in \mathbb{N}} \sum_{k \in K_{2}}\left|a_{n k} M^{-1}\right|^{p_{k}^{\prime}}<\infty, \\
& \exists\left(\alpha_{k}\right), \quad \lim _{n \rightarrow \infty}\left|a_{n k}-\alpha_{k}\right|^{q_{n}}=0 \quad \text { for all } k, \\
& \exists\left(\alpha_{k}\right), \forall L, \quad \sup _{n \in \mathbb{N}} \sup _{k \in K_{1}}\left(\left|a_{n k}-\alpha_{k}\right| L^{1 / q_{n}}\right)^{p_{k}}<\infty, \\
& \exists\left(\alpha_{k}\right), \forall L, \exists M, \sup _{n \in \mathbb{N}}\left(\left|a_{n k}-\alpha_{k}\right| L^{1 / q_{n}} M^{-1}\right)^{p_{k}^{\prime}}<\infty, \\
& \exists M, \quad \sup _{k \in \mathbb{N}} \sum_{k \in K_{2}}\left|a_{n k} M^{-1}\right|^{p_{k}}<\infty, \\
& \exists M, \quad \sup _{n \in \mathbb{N}} \sum_{k}\left|a_{n k}\right| M^{-1 / p_{k}}<\infty, \\
& \exists \alpha, \quad \lim _{n \rightarrow \infty}\left|\sum_{k} a_{n k}-\alpha_{k}\right|<\infty, \\
& \forall M, \quad \sup _{n \in \mathbb{N}} \sum_{k}\left|a_{n k}\right| M^{1 / p_{k}}<\infty \\
& \exists\left(\alpha_{k}\right), \exists M, \lim _{n \rightarrow \infty}\left(\sum_{k}\left|a_{n k}-\alpha_{k}\right| M^{-1 / p_{n}}\right)=0, \\
& \exists L, \quad \sup _{n \in \mathbb{N}} \sup _{k \in K_{1}}\left|a_{n k} L^{-1 / q_{n}}\right|^{p_{k}}<\infty, \\
& \exists L, \quad \sup _{n \in \mathbb{N}} \sum_{k \in K_{2}}\left|a_{n k} L^{-1 / q_{n}}\right|^{p_{k}}<\infty \text {, } \\
& \exists M, \quad \sup _{n \in \mathbb{N}}\left(\sum_{k \in K}\left|a_{n k}\right| M^{-1 / p_{k}}\right)^{q_{n}}<\infty, \\
& \sup _{n \in \mathbb{N}}\left|\sum_{k} a_{n k}\right|^{q_{n}}<\infty \\
& \forall M, \quad \sup _{n \in \mathbb{N}}\left(\sum_{k}\left|a_{n k}\right| M^{1 / p_{k}}\right)^{q_{n}}<\infty .
\end{aligned}
$$

Corollary 3.12. (i) $A=\left(a_{n k}\right) \in\left(c_{0}(u, v, p ; B): \ell(q)\right)$ if and only if (3.20) holds with $\bar{a}_{n k}$ instead of $a_{n k}$ and (3.18) also holds with $\mu=c_{0}$.

(ii) $A=\left(a_{n k}\right) \in\left(c_{0}(u, v, p ; B): c(q)\right)$ if and only if (3.31), (3.34) and (3.35) hold with $\bar{a}_{n k}$ instead of $a_{n k}$ and (3.18) also holds with $\mu=c_{0}$.

(iii) $A=\left(a_{n k}\right) \in\left(c_{0}(u, v, p ; B): \ell(q)\right)$ if and only if (3.41) holds with $\bar{a}_{n k}$ instead of $a_{n k}$ and (3.18) also holds with $\mu=c_{0}$.

Corollary 3.13. (i) $A=\left(a_{n k}\right) \in(c(u, v, p ; B): \ell(q))$ if and only if (3.20) and (3.21) hold with $\bar{a}_{n k}$ instead of $a_{n k}$ and (3.18) also holds with $\mu=c$.

(ii) $A=\left(a_{n k}\right) \in(c(u, v, p ; B): c(q))$ if and only if (3.31), (3.34)-(3.36) hold with $\bar{a}_{n k}$ instead of $a_{n k}$ and (3.18) also holds with $\mu=c$. 
(iii) $A=\left(a_{n k}\right) \in(c(u, v, p ; B): \ell(q))$ if and only if (3.41) and (3.42) hold with $\bar{a}_{n k}$ instead of $a_{n k}$ and (3.18) also holds with $\mu=c$.

Corollary 3.14. $(i) A=\left(a_{n k}\right) \in\left(\ell_{\infty}(u, v, p ; B): \ell(q)\right)$ if and only if (3.22) holds with $\bar{a}_{n k}$ instead of $a_{n k}$ and (3.18) also holds with $\mu=\ell_{\infty}$.

(ii) $A=\left(a_{n k}\right) \in\left(\ell_{\infty}(u, v, p ; B): c(q)\right)$ if and only if (3.28) hold with $\bar{a}_{n k}$ instead of $a_{n k}$ and (3.18) also holds with $\mu=\ell_{\infty}$.

(iii) $A=\left(a_{n k}\right) \in\left(\ell_{\infty}(u, v, p ; B): \ell(q)\right)$ if and only if (3.37) and (3.38) hold with $\bar{a}_{n k}$ instead of $a_{n k}$ and (3.18) also holds with $\mu=\ell_{\infty}$.

(iv) $A=\left(a_{n k}\right) \in\left(\ell_{\infty}(u, v, p ; B): \ell_{\infty}(q)\right)$ if and only if (3.43) holds with $\bar{a}_{n k}$ instead of $a_{n k}$ and (3.18) also holds with $\mu=\ell_{\infty}$.

Corollary 3.15. (i) $A=\left(a_{n k}\right) \in\left(c_{0}(p): c(u, v, p ; B)\right)$ if and only if (3.31), (3.34) and (3.35) hold with $b_{n k}$ instead of $a_{n k}$.

(ii) $A=\left(a_{n k}\right) \in\left(c_{0}(p): \ell_{\infty}(u, v, p ; B)\right)$ if and only if (3.41) holds with $b_{n k}$ instead of $a_{n k}$.

Corollary 3.16. (i) $A=\left(a_{n k}\right) \in(c(p): c(u, v, p ; B))$ if and only if (3.31), (3.34)-(3.36) hold with $b_{n k}$ instead of $a_{n k}$.

(ii) $A=\left(a_{n k}\right) \in\left(c(p): \ell_{\infty}(u, v, p ; B)\right)$ if and only if (3.41) and (3.42) hold with $b_{n k}$ instead of $a_{n k}$.

Corollary 3.17. (i) $A=\left(a_{n k}\right) \in\left(\ell_{\infty}(p): c_{0}(u, v, p ; B)\right)$ if and only if (3.28) holds with $b_{n k}$ instead of $a_{n k}$.

(ii) $A=\left(a_{n k}\right) \in\left(\ell_{\infty}(p): \ell_{\infty}(u, v, p ; B)\right)$ if and only if (3.43) holds with $b_{n k}$ instead of $a_{n k}$.

(iii) $A=\left(a_{n k}\right) \in\left(\ell_{\infty}(p): c(u, v, p ; B)\right)$ if and only if (3.37) and (3.38) hold and $b_{n k}$ instead of $a_{n k}$.

Corollary 3.18. (i) $A=\left(a_{n k}\right) \in\left(\ell(p): c_{0}(u, v, p ; B)\right)$ if and only if (3.23) and (3.25) hold with $b_{n k}$ instead of $a_{n k}$.

(ii) $A=\left(a_{n k}\right) \in(\ell(p): c(u, v, p ; B))$ if and only if (3.29) and (3.33) hold with $b_{n k}$ instead of $a_{n k}$.

(iii) $A=\left(a_{n k}\right) \in\left(\ell(p): \ell_{\infty}(u, v, p ; B)\right)$ if and only if (3.39) and (3.40) hold and $b_{n k}$ instead of $a_{n k}$.

\section{COMPACTNESS OF MATRIX OPERATORS}

In the section, we establish some identities or estimates for the operator norms and the Hausdorff measures of noncompactness of certain matrix operators on the spaces $c_{0}(u, v ; B)$ and $\ell_{\infty}(u, v, ; B)$. Further, by using the Hausdorff measure of noncompactness, we characterize certain some classes of compact operators on these spaces. It is quite natural to find condition for a matrix map between 
$B K$-space to define a compact operator since a matrix transformation between $B K$-spaces are continuous. This can be achieved by applying the Hausdorff measure of noncompactness. Recently, several authors characterized classes of compact operators given by infinite matrices on some sequence spaces by using this method. For example, in [20, 21], Mursaleen and Noman, Malkowsky and Rakočević [10], Djolović and Malkowsky [30], and Karaisa [8] established some identities or estimate for the operator norms and Hausdorff measure of noncompactness of the linear operator given by infinite matrices that map an arbitrary $B K$-space or the matrix domain of triangles in arbitrary $B K$-space. Further, They characterized some classes of compact operators on these spaces by using the Hausdorff measure of noncompactness.

4.1. Notations and Auxiliary Facts. Now, we give some related definitions, notations and preliminary result.

Let $X$ and $Y$ be Banach space. Then, we write $\mathcal{B}(X, Y)$ for the set of all bounded linear operators $L: X \longrightarrow Y$, which is a Banach space with the operator norm given by $\|L\|=\sup _{x \in S_{X}}\|L(x)\|_{Y}$ for all $L \in \mathcal{B}(X, Y)$, where $S_{X}$ denotes the unit sphere in $X$, the sequence $\left(L\left(x_{n}\right)\right)$ has a subsequence which converges in $Y$. By $\mathcal{C}(X, Y)$, we denote the class of all compact operator in $\mathcal{B}(X, Y)$. An operator $L \in \mathcal{B}(X, Y)$ is said to be of finite rank if $\operatorname{dim} R(L)<\infty$, where $R(L)$ denotes range of $L$. An operator of finite rank is clearly compact.

If $(\|\cdot\|, X)$ is a normed sequence space then we write

$$
\|a\|_{X}^{*}=\sup _{x \in S_{X}} \sum_{k=0}^{\infty}\left|a_{k} x_{k}\right|
$$

for $a \in w$ provided the expression on the right-hand side exists and is finite which the case whenever $X$ is a $B K$-space and $a \in X^{\beta}$ [11]. Let $S$ and $M$ be subsets of metric space $(X, d)$ and $\varepsilon>0$. Then $S$ is called an $\varepsilon$-net of $M$ in $X$ if every $x \in M$ there exists $s \in S$ such that $d(x, s)<\varepsilon$. Further the set $S$ is finite, then the $\varepsilon$-net $S$ of $M$ is called a finite $\varepsilon$-net of $M$, and we say that $M$ has a finite $\varepsilon$-net in $X$. A subset of a metric space is said to be totally bounded if it has a finite $\varepsilon$-net for every $\varepsilon>0$. By $\mathcal{M}_{X}$, we denote the collection of all bounded subsets of a metric space $(X, d)$. If $Q \in \mathcal{M}_{X}$, then the Hausdorff measure of noncompactness of the set $Q$, denotes by $\chi(Q)$, is defined by

$$
\chi(Q)=\inf \{\varepsilon>0: Q \text { has a finite } \varepsilon \text {-net in } X\} .
$$

The function $\chi: \mathcal{M}_{X} \longrightarrow[0, \infty)$ is called the Hausdorff measure of noncompactness [11, p. 387].

The basic properties of the Hausdorff measure of noncompactness can be found in [12, Lemma 2]; for example if $Q, Q_{1}$ and $Q_{2}$ are bounded subsets of a metric space $(X, d)$, then

$$
\begin{aligned}
& \chi(Q)=0 \text { if and only if } Q \text { is totally bounded, } \\
& Q_{1} \subset Q_{2} \text { implies } \chi\left(Q_{1}\right) \leq \chi\left(Q_{2}\right) .
\end{aligned}
$$


Further, if $X$ is a normed space, then the function $\chi$ has some additional properties connected with the linear structure, that is

$$
\begin{aligned}
& \chi\left(Q_{1}+Q_{2}\right) \leq \chi\left(Q_{1}\right)+\chi\left(Q_{2}\right), \\
& \chi(\alpha Q)=|\alpha| \chi(Q) \text { for all } \alpha \in \mathbb{C} .
\end{aligned}
$$

We shall need the following known results for our investigation.

Lemma 4.1. [12, Lemma 15(a)] Let $\varphi \supset X$ and $Y$ be a BK-space. Then, we have $(X, Y) \subset \mathcal{B}(X, Y)$, that is, every matrix $A \in(X, Y)$ defines an operator $L_{A} \in \mathcal{B}(X, Y)$ by $L_{A}(x)=$ Ax for all $x \in X$.

Lemma 4.2. [13, Theorem 3.8)] Let $T$ be a triangle. Then, we have

(a) For arbitrary subsets $X$ and $Y$ of $\omega, A \in\left(X, Y_{T}\right)$ if and only if $B=T A \in$ $(X, Y)$.

(b) Further, if $X$ and $Y$ are $B K$ spaces and $A \in\left(X, Y_{T}\right)$, then $\left\|L_{A}\right\|=\left\|L_{B}\right\|$.

Lemma 4.3. [31, Lemma 5.2] Let $\varphi \supset X$ be a BK space and $Y$ be any of the spaces $c_{0}, c$ or $\ell_{\infty}$. If $A \in(X, Y)$, then we have

$$
\left\|L_{A}\right\|=\|A\|_{\left(X, \ell_{\infty}\right)}=\sup _{n}\left|A_{n}\right|_{X}^{*}<\infty .
$$

Lemma 4.4. [13, Theorem 1.29] Let $X$ be any of the spaces $c, c_{0}$ or $\ell_{\infty}$. Then, we have $X^{\beta}=\ell_{1}$ and $\|a\|_{X}^{*}=\|a\|_{\ell_{1}}$ for all $a \in \ell_{1}$.

Lemma 4.5. Let $X$ be denote any of the spaces $c_{0}(u, v ; B)$ and $\ell_{\infty}(u, v ; B)$. If $a=\left(a_{k}\right) \in X^{\beta}$, then we $\widetilde{a}=\left(\widetilde{a}_{k}\right) \in \ell_{1}$ and equality

$$
\sum_{k=0}^{\infty} a_{k} x_{k}=\sum_{k=0}^{\infty} \widetilde{a}_{k} y_{k}
$$

holds for every $x=\left(x_{k}\right) \in X$, where $y=\widehat{G}(x)$ is the associated sequence defined by (3.1) and

$$
\widetilde{a}_{k}=\frac{1}{u_{k}}\left[\frac{a_{k}}{r v_{k}}+\left(\frac{1}{r v_{k}}+\frac{1}{s v_{k+1}}\right) \sum_{j=k+1}^{\infty}\left(\frac{-s}{r}\right)^{k-j} a_{j}\right] .
$$

Theorem 4.6. Let $X$ be denote any of the spaces $c_{0}(u, v ; B)$ and $\ell_{\infty}(u, v ; B)$. Then, we have

$$
\|a\|_{X}=\|\widetilde{a}\|_{\ell_{1}}=\sum_{k=0}^{\infty}\left|\widetilde{a}_{k}\right|<\infty .
$$

for all $a=\left(a_{k}\right) \in X^{\beta}$, where $\widetilde{a}=\left(\widetilde{a}_{k}\right)$ is as in Lemma 4.5.

Proof. Let $Y$ be the respective one of the space $c_{0}$ or $\ell_{\infty}$, and take any $a=\left(a_{k}\right) \in$ $X^{\beta}$. Then, we have by Lemma 4.5 that $\widetilde{a}=\left(\widetilde{a}_{k}\right) \in \ell_{1}$ and the equality (4.2) holds for all sequences $x=\left(x_{k}\right) \in X$ and $y=\left(y_{k}\right) \in Y$ which are connected by the relation (3.1). Further, it follows by (3.2) that $x \in S_{X}$ if and only if $y \in S_{Y}$. Therefore, we derive from (4.1) and (4.2) that

$$
\|a\|_{X}=\sup _{x \in S_{X}}\left|\sum_{k=0}^{\infty} a_{k} x_{k}\right|=\sup _{y \in S_{Y}}\left|\sum_{k=0}^{\infty} \widetilde{a}_{k} y_{k}\right|=\|\widetilde{a}\|_{Y} .
$$


Since $\widetilde{a} \in \ell_{1}$, we obtain from Lemma 4.4 that

$$
\|a\|_{X}^{*}=\|\widetilde{a}\|_{Y}^{*}=\|\widetilde{a}\|_{\ell_{1}}^{*}<\infty .
$$

Lemma 4.7. Let $X$ be any of the space $c_{0}(u, v ; B)$ or $\ell_{\infty}(u, v ; B), Y$ the respective one of the spaces $c_{0}$ or $\ell_{\infty}, Z$ a sequence space and $A=\left(a_{n k}\right)$ an infinite matrix. If $A \in(X, Z)$, then $\bar{A} \in(Y, Z)$ such that $A x=\bar{A} y$ for all sequences $x \in X$ and $y \in Y$ which are connected by the relation (3.1), where $\bar{A}=\left(\bar{a}_{n k}\right)$ is the associated matrix defined by (3.17).

Proof. It can be similarly proved by the same technique in [21, Lemma 2.3].

Lemma 4.8. Let $X$ be any of the space $c_{0}(u, v ; B)$ or $\ell_{\infty}(u, v ; B), A=\left(a_{n k}\right)$ an infinite matrix and $\bar{A}=\left(\bar{a}_{n k}\right)$ is the associated matrix. If $A$ is in any of the classes $\left(X, c_{0}\right),(X, c)$ or $\left(X, \ell_{\infty}\right)$, then

$$
\left\|L_{A}\right\|=\|A\|_{\left(X, \ell_{\infty}\right)}=\sup _{n}\left(\sum_{n}^{\infty}\left|\bar{a}_{n k}\right|\right)<\infty
$$

Proof. This is immediate by combining Lemmas 4.3 and 4.6.

The following results shows how to compute the Hausdorff measure of noncompactness in the space $c_{0}$.

Lemma 4.9. [29, Theorem 3.3] Let $Q \in \mathcal{M}_{c_{0}}$ and $p_{r}: c_{0} \longrightarrow c_{0}(r \in \mathbb{N})$ be operator defined by $p_{r}(x)=\left(x_{0}, x_{1}, \ldots, x_{r}, 0,0, \ldots\right)$ for all $x=\left(x_{k}\right) \in c_{0}$. Then we have

$$
\chi(Q)=\lim _{r \rightarrow \infty}\left(\sup _{x \in Q}\left\|\left(I-p_{r}\right)(x)\right\|_{\ell_{\infty}}\right),
$$

where $I$ is the identity operator on $c_{0}$.

Further, we know by [13, Theorem 1.10] that every $z=\left(z_{k}\right) \in$ c has a unique representation $z=\bar{z} e+\sum_{n}^{\infty}\left(z_{n}-\bar{z}\right) e^{(n)}$, where $\bar{z}=\lim _{n \rightarrow \infty} z_{n}$. Thus, we define the projectors $p_{r}: c \longrightarrow c(r \in \mathbb{N})$ by

$$
p_{r}=\bar{z} e+\sum_{n=0}^{r}\left(z_{n}-\bar{z}\right) e^{(n)} ; \quad(r \in \mathbb{N})
$$

for all $z=\left(z_{k}\right) \in c$ with $\bar{z}=\lim _{n \rightarrow \infty} z_{n}$. In this situation, the following result gives an estimate for the Hausdorff measure of noncompactness in the space $c$.

Lemma 4.10. [12, Theorem 5(b)] Let $Q \in \mathcal{M}_{c}$ and $p_{r}: c \longrightarrow c(r \in \mathbb{N})$ be the projector onto the linear span of $\left(e^{(0)}, e^{(1)}, \ldots, e^{(r)}\right)$. Then, we have

$$
\frac{1}{2} \lim _{r \rightarrow \infty}\left(\sup _{x \in Q}\left\|\left(I-p_{r}\right)(x)\right\|_{\ell_{\infty}}\right) \leq \chi(Q) \leq \lim _{r \rightarrow \infty}\left(\sup _{x \in Q}\left\|\left(I-p_{r}\right)(x)\right\|_{\ell_{\infty}}\right),
$$

where $I$ is the identity operator on $c$.

The next lemma is related to the Hausdorff measure of noncompactness of a bounded linear operator. 
Lemma 4.11. [13, Theorem 2.25] Let $X$ and $Y$ be Banach spaces and $L \in$ $B(X, Y)$. Then, we have

$$
\left\|L_{A}\right\|_{\chi}=\chi\left(L\left(S_{X}\right)\right)
$$

and

$$
L \in \mathcal{C}(X, Y) \text { if and only if }\left\|L_{A}\right\|_{\chi}=0 .
$$

4.2. Compact Operators on the Spaces $c_{0}(u, v ; B)$ and $\ell_{\infty}(u, v ; B)$. In this part, we establish some identities or estimates for the Hausdorff measures of noncompactness of certain matrix operators on the spaces $c_{0}(u, v ; B)$ and $\ell_{\infty}(u, v ; B)$. Further, we apply our results to characterize some classes of compact operators on those spaces. We begin with the following lemmas which will be used in proving our results.

Lemma 4.12. [20, Lemma 3.1] Let $X$ be any of the space $c_{0}$ or $\ell_{\infty}$. If $A \in(X, c)$

$$
\begin{aligned}
& \alpha_{k}=\lim _{n \rightarrow \infty} a_{n k} \text { exists for every } k \in \mathbb{N}, \\
& \alpha=\left(\alpha_{k}\right) \in \ell_{1}, \\
& \sup _{n}\left(\sum_{k}^{\infty}\left|a_{n k}-\alpha_{k}\right|\right)<\infty, \\
& \lim _{n \rightarrow \infty} A_{n}=\sum_{k}^{\infty} \alpha_{k} x_{k} \text { for all } x=\left(x_{k}\right) \in X .
\end{aligned}
$$

Lemma 4.13. [23, Theorem 3.7] Let $X \supset \phi$ be a BK-space. Then we have (a) If $A \in\left(X, c_{0}\right)$, then

$$
\left\|L_{A}\right\|_{\chi}=\limsup _{n \rightarrow \infty}\left\|A_{n}\right\|_{X}^{*}
$$

(b) If $A \in\left(X, \ell_{\infty}\right)$, then

$$
0 \leq\left\|L_{A}\right\|_{\chi} \leq \limsup _{n \rightarrow \infty}\left\|A_{n}\right\|_{X}^{*}
$$

Theorem 4.14. Let $X$ denote any of the spaces $c_{0}(u, v ; B)$ and $\ell_{\infty}(u, v ; B)$. Then we have

(a) If $A \in\left(X, c_{0}\right)$, then

$$
\left\|L_{A}\right\|_{\chi}=\limsup _{n \rightarrow \infty}\left(\sum_{k=0}^{\infty}\left|\bar{a}_{n k}\right|\right)
$$

and

$$
L_{A} \quad \text { compact if and only if } \lim _{n \rightarrow \infty}\left(\sum_{k=0}^{\infty}\left|\bar{a}_{n k}\right|\right)=0 \text {. }
$$


(b) If $A \in\left(X, \ell_{\infty}\right)$, then

$$
0 \leq\left\|L_{A}\right\|_{\chi} \leq \limsup _{n \rightarrow \infty}\left(\sum_{k=0}^{\infty}\left|\bar{a}_{n k}\right|\right)
$$

and

$$
L_{A} \quad \text { compact if and only if } \lim _{n \rightarrow \infty}\left(\sum_{k=0}^{\infty}\left|\bar{a}_{n k}\right|\right)=0 .
$$

Proof. Let $A \in\left(X, c_{0}\right)$. Since $A_{n} \in X^{\beta}$ for all $n \in \mathbb{N}$, we have from Lemma 4.6 that

$$
\left\|A_{n}\right\|_{X}=\left\|\bar{A}_{n}\right\|_{\ell_{1}}=\sum_{k=0}^{\infty}\left|\bar{a}_{n k}\right|<\infty .
$$

Thus, we get (4.7) from (4.9), (4.3) and Lemma 4.13(a). We derived (4.8) from (4.6). Part (b) can be proved similarly by using Lemma 4.13(b).

Theorem 4.15. Let $X$ denote any of the spaces $c_{0}(u, v ; B)$ and $\ell_{\infty}(u, v ; B)$. If $A \in(X, c)$, then we have

$$
\frac{1}{2} \limsup _{n \rightarrow \infty}\left(\sum_{k=0}^{\infty}\left|\bar{a}_{n k}-\bar{\alpha}_{k}\right|\right) \leq\left\|L_{A}\right\|_{\chi} \leq \limsup _{n \rightarrow \infty}\left(\sum_{k=0}^{\infty}\left|\bar{a}_{n k}-\bar{\alpha}_{k}\right|\right)
$$

and

$$
L_{A} \quad \text { compact if and only if } \lim _{n \rightarrow \infty}\left(\sum_{k=0}^{\infty}\left|\bar{a}_{n k}-\bar{\alpha}_{k}\right|\right)=0 \text {, }
$$

where $\lim _{n \rightarrow \infty} \bar{a}_{n k}=\bar{\alpha}_{k}$.

Proof. By combining Lemma 4.7 and Lemma 4.12, we deduce that the expression in (4.7) exists. We write $S=S_{X}$, for short. Then, we obtain by (4.5) and Lemma 4.1 that

$$
\left\|L_{A}\right\|_{\chi}=\chi(A S)
$$

which means $A S \in \mathcal{M}_{c}$, where is the class of all bounded subsets of $c$. Then, we are going to apply Lemma 4.10 to get an estimate for the value of $\chi(A S)$ in (4.12). For this, let $p_{r}: c \longrightarrow c$ be the projectors defined by (4.4). Then, we have for every $r \in \mathbb{N}$ that $\left(I-p_{r}\right)(z)=\sum_{n=r+1}^{\infty}\left(z_{n}-z\right) e^{n}$ and hence,

$$
\left\|\left(I-p_{r}\right)(z)\right\|_{\ell_{\infty}}=\sup _{n>r}|z-\bar{z}|
$$

for all $z \in c$. Thus, from (4.12) and Lemma 4.10 that

$$
\frac{1}{2} \lim _{r \rightarrow \infty}\left(\sup _{x \in S}\left\|\left(I-p_{r}\right)(A x)\right\|_{\ell_{\infty}}\right) \leq\left\|L_{A}\right\|_{\chi} \leq \lim _{r \rightarrow \infty}\left(\sup _{x \in S}\left\|\left(I-p_{r}\right)(A x)\right\|_{\ell_{\infty}}\right)
$$

Now, for every given $x \in X$ and $y \in Y$ be associated sequence defined by (3.1), where $Y$ be the respective one of the space $c_{0}$ or $\ell_{\infty}$. Since $A \in(X, c)$, we have by Lemma 4.7 that $\bar{A} \in(Y, c)$ and $A x=\bar{A} y$. Further, it follows from Lemma 
4.12 that the limits $\bar{\alpha}_{k}=\lim _{n \rightarrow \infty} \bar{a}_{n k}$ exists for all $k, \bar{\alpha}=\left(\bar{\alpha}_{k}\right) \in \ell_{1}=Y^{\beta}$ and $\lim _{n \rightarrow \infty}(\bar{A} y)_{n}=\sum_{k=0}^{\infty} \bar{\alpha}_{k} y_{k}$. Thus, we derive from (4.13) that

$$
\begin{aligned}
\left\|\left(I-p_{r}\right)(A x)\right\|_{\ell_{\infty}} & =\left\|\left(I-p_{r}\right)(\bar{A} y)\right\|_{\ell_{\infty}} \\
& =\sup _{n>r}\left|\bar{A}_{n}(y)-\sum_{k=0}^{\infty} \bar{\alpha}_{k} y_{k}\right| \\
& =\sup _{n>r}\left|\sum_{k=0}^{\infty}\left(\bar{a}_{n k}-\bar{\alpha}_{k}\right) y_{k}\right|
\end{aligned}
$$

for $r \in \mathbb{N}$. Furthermore, since $x \in S=S_{X}$ if and only if $y \in S_{Y}$, we obtain by (4.1) and Lemma 4.1

$$
\begin{aligned}
\sup _{X \in S}\left\|\left(I-p_{r}\right)(A x)\right\|_{\ell_{\infty}} & =\sup _{n>r}\left(\sup _{Y \in S_{Y}}\left|\sum\left(\bar{a}_{n k}-\bar{\alpha}_{k}\right) y_{k}\right|\right) \\
& =\sup _{n>r}\left\|\bar{A}_{n}-\bar{\alpha}\right\|_{Y}^{*} \\
& =\sup _{n>r}\left\|\bar{A}_{n}-\bar{\alpha}\right\|_{\ell_{1}}
\end{aligned}
$$

for all $r \in \mathbb{N}$. Thus, we get (4.10) and (4.11) from (4.14) and (4.6), respectively and this concludes the proof.

Acknowledgement. We thank the referees for their careful reading of the original manuscript and for the valuable comments.

\section{REFERENCES}

1. A. Sönmez and F. Başar, Generalized Diffrence space on none-absolute type of convergent and null sequences, Abstr. Appl. Anal. 2012 Article ID 435076 (2012) 1-20.

2. A. Wilansky, Summability through Functional Analysis, North-Holland Mathematics Studies 85, Amsterdam-New York-Oxford, 1984.

3. S. Demiriz and C. Çakan, Some new paranormed difference sequence spaces and weighted core, Comput. Math. Appl. 64 (2012) 1726-1739.

4. B. Altay and F. Başar, On the paranormed Riesz sequence spaces of non-absolute type, Southeast Asian Bull. Math. 26(5) (2003) 701-715

5. B. Choudhary and S.K. Mishra, On Köthe-Toeplitz duals of certain sequence spaces and their matrix transformations, Indian J. Pure Appl. Math. 24 (1993) , no. 5, 291-301.

6. F. Başar, Summability Theory and Its Applications, Bentham Science Publishers, e-books, Monographs İstanbul, 2012.

7. F. Başar and A. Karaisa, Some new generalized difference spaces of nonabsolute type derived from the spaces $\ell_{p}$ and $\ell_{\infty}$, The Scientific World Journal 2013 Article ID 3493462013 doi:10.1155/2013/349346 1-15.

8. A. Karaisa, Hausdorff measure of noncompactness in some sequence space of a triple band matrix J. Inequal. App. 2013, 2013:503, 11 pp.

9. C. Aydın and F. Başar, Some new diference sequence spaces, Appl. Math. Comput. 154(2004) 677-693.

10. E. Malkowsky and V. Rakočević, On matrix domains of triangles, Appl. Math. Comput. 189, no. 2, (2007) 1146-1163.

11. E. Malkowsky and V. Rakočević and S. Živkovi ć, Matrix transformations between the sequence spaces $w_{0}^{p}(\Lambda), v_{0}^{p}(\Lambda), c_{0}^{p}(\Lambda)(1<p<\infty)$ and certain BK spaces, Appl. Math. Comput 147 (2004), no. 2, 377-396 . 
12. E.Malkowsky, Compact matrix operators between some BK-spaces in:M.Mursaleen (Ed.), Modern Methods of Analysis and its Applications, Anamaya Publ., New Delhi, (2010) 86-120.

13. E. Malkowsky and V. Rakočević, An introduction into the theory of sequence spaces and measures of noncompactness Zbornik Mat. institut sanu (Beograd)radova 9 (2000), no. 17, 143-34.

14. F. Başar and B. Altay, Some paranormed sequence spaces of non-absolute type derived by weighted mean J. Math. Anal. Appl 319 (2006) , no. 2, 494-508.

15. H. Nakano, Modulared sequence spaces Proc. Japan Acad. 27 (1951) 508-512.

16. K.G. Grosse-Erdmann, Matrix transformations between the sequence spaces of Maddox J. Math. Anal. Appl. 180(1993) 223-238.

17. F. Özger and F. Başar, Domain of the double sequential band matrix $B(r, s)$ on some Maddox's spaces, Acta Math.Sci. 34 (2014), no. 2, 394-408.

18. M. Başarır, On the generalized Riesz B-difference sequence spaces Filomat 24 (2010), no. $4,35-52$.

19. E. Malkowsky and F. Özger, A note on some sequence spaces of weighted means, Filomat 26 (2012), no. 3, 511-518.

20. M. Mursaleen and A.K. Noman, Applications of the Hausdorff measure of noncompactness in some sequence spaces of weighted means Comput. Math. Appl. 60 (2010) 1245-125.

21. M. Mursaleen and A.K. Noman, Compactness of matrix operators on some new difference sequence spaces, Linear Algebra Appl. 436 (2012) 41-52.

22. A.M. Jarrah and E. Malkowsky, BK spaces, bases and linear operators Rendiconti Circ. Mat. Palermo II 52 177-191 (1990).

23. M. Mursaleen and A.K. Noman, Compactness by the Hausdorff measure of noncompactness Nonlinear Anal. 73 (2010) 2541-2557.

24. M. Mursaleen and A.K. Noman, On some new difference sequence spaces of non-absolute type Math. Comput. Modelling 52 (2010) 603-617.

25. I.J. Maddox, Elements of Functional Analysis, 2nd ed. The University Press Cambridge, (1988).

26. J. Boos, Oxford University Press Inc, New York Classical and Modern Methods in Summability Oxford University Press Inc, New York 2000.

27. S. Simons, The sequence spaces $\ell(p v)$ and $m(p v)$ Proc. London Math. Soc. (3) 15 (1965), no. $1,422-36$.

28. A. Karaisa and Ü. Karabiyık, Almost sequence spaces derived by the domain of the matrix, Abstr. Appl. Anal. 2013 Article ID 783731 (2013) 1-8.

29. I. Djolović and E. Malkowsky, A note on Fredholm operators on $\left(c_{0}\right) T$, Appl. Math. Lett.11 (2009) 1734-1739.

30. I. Djolović and E. Malkowsky, A note on compact operators on matrix domains, J. Math. Anal. Appl. 340(2008), no. 1, 291-303.

31. I. Djolović and E. Malkowsky, Matrix transformations and compact operators on some new mth-order difference sequences, Appl. Math. Comput. 198, no. 2, (2008) 700-714.

32. I.J. Maddox, Paranormed sequence spaces generated by infinite matrices, Proc. Camb. Phil. Soc. 64(1968) 335-340.

33. V. Karakaya, A.K. Noman and H. Polat, On paranormed $\lambda$-sequence spaces of non-absolute type, Math. Comput. Modelling 54 (2011) 1473-480.

Department of Mathematics-Computer Science, Necmettin Erbakan UniverSity, Meram Campus, 42090 Meram, Konya, Turkey.

E-mail address: alikaraisa@hotmail.com; akaraisa@konya.edu.tr 